\title{
SZEGÖ KERNEL FOR HARDY SPACE OF MATRIX FUNCTIONS *
}

\author{
Fuli HE \\ School of Mathematics and Statistics, Central South University, Changsha 410083, China \\ E-mail: hefuli999@163.com \\ Min $K U^{\dagger}$ \\ CIDMA, Department of Mathematics, University of Aveiro, Portugal \\ E-mail:kumin0844@163.com \\ Uwe KÄHLER \\ CIDMA, Department of Mathematics, University of Aveiro, Portugal \\ E-mail: ukaehler@ua.pt
}

\begin{abstract}
By the characterization of the matrix Hilbert transform in the Hermitian Clifford analysis, we introduce the matrix Szegö projection operator for the Hardy space of Hermitean monogenic functions defined on a bounded sub-domain of even dimensional Euclidean space, establish the Kerzman-Stein formula which closely connects the matrix Szegö projection operator with the Hardy projection operator onto the Hardy space, and get the matrix Szegö projection operator in terms of the Hardy projection operator and its adjoint. Furthermore, we construct the explicit matrix Szegö kernel function for the Hardy space on the sphere as an example, and get the solution to a boundary value problem for matrix functions.
\end{abstract}

Key words Hardy space; Hermitean Clifford analysis; Szegö projection; matrix function

2010 MR Subject Classification 30G35; 15A66; 30C40; 31A25; 31B10

\section{Introduction}

The Szegö kernel function (for short Szegö kernel) and the Szegö projection, which were first introduced by the Hungarian mathematician Gábor Szegö in 1921 (see Ref. [1]), played an important role in the development of the complex analysis. The Szegö kernel is a reproducing kernel for the Hilbert space of all square integrable holomorphic functions defined on a domain (see Refs. e.g. $[2,3]$ ), which is of importance to reveal the properties of the holomorphic map between two domains and to solve the boundary value problems. For instance, the conformal

*The project is supported by Portuguese funds through the CIDMA Center for Research and Development in Mathematics and Applications, and the Portuguese Foundation for Science and Technology ("FCT-Fundação para a Ciência e a Tecnologia") within project PEst- OE/MAT/UI4106/2014 and by NNSF of China(Grant No.11371375,11301547). The corresponding author is the recipient of a Postdoctoral Foundation from FCT under Grant No. SFRH/BPD/74581/2010.

${ }^{\dagger}$ Corresponding author: Min KU 
mappings onto the canonical domains, the classical functions, and other important objects of potential theory can be simply expressed in virtue of the Szegö kernels (seen in Refs. e.g. [3-5]). The Szegö projection operator associates with smooth boundary of a domain is of fundamental interest in the complex analysis. Its action can often be expressed as an integration against a distribution, known as the Szegö kernel, and the study of its is naturally introduced for the space of square integrable function onto Hardy space defined on the boundaries of a domain (see Refs. e.g. $[1,3])$. This allows us to deeper understand the geometric and functional analytic properties in the complex analysis and the harmonic analysis (see Refs. e.g. [2, 3]).

However, it is not feasible to obtain the closed formulas of the Szegö kernels for the general domains of the complex plane. One of the difficulties lies in that one could not obtain an estimation for these kernels in terms of the geometrical properties of the considered domains. Only for the special cases, including the unit circle, the Szegö kernels can be computed explicitly. Moreover, it is showed by Kerzman and Stein in Ref. [2] that the unit circle is the only planar region, for which the Szegö kernel and the Cauchy kernel coincide. On the contrary, for the general domains on the complex plane, they established the well-known Kerzman-Stein formula (see Refs. e.g. [2, 3]), which connects the Szegö projection with the Hardy projection. Later on, in Refs. [6-8], Bernstein, Calderbank, Delanghe and their collaborators generalized the Kerzman-Stein formula to the higher dimensions, making full use of the methods of the orthogonal Clifford analysis, seen in Refs. e.g. [12-19]. More related results on the Szegö kernel and the Szegö projection in the orthogonal Clifford analysis can be also found in Refs. e.g. $[9-11]$.

Offering a refinement of the orthogonal case, the Hermitean Clifford analysis, seen in Refs. e.g. [20-24], emerged as a new and successful branch of Clifford analysis. It focuses on the simultaneous null-solutions of the two complex Hermitean Dirac operators, which recently invokes new tools for the study of circulant $(2 \times 2)$ matrix functions. A Cauchy integral formula in the Hermitean Clifford was constructed in terms of circulant $(2 \times 2)$ matrix functions (see Refs. e.g. [25-28]), and the relationship with holomorphic function theory of several complex variables was observed, seen in Refs. e.g. [24-26]. The Hermitean Cauchy transform, which gave rise to the Hardy projection to be skew, and the related decomposition problems of continuous functions were discussed in Refs. [27, 28]. The new Hilbert-like matrix operator was revealed by the non-tangential boundary limits of the Hermitean Cauchy transform in Refs. e.g. $[25,26]$. Much recent progress can be also seen in Refs. $[29,30]$ or elsewhere. Under this setting it is natural for us to want to know what is the matricial Szegö projection. But, to our knowledge, little attention has been devoted to up to now. This leads us to further consider the Hardy space for circulant $(2 \times 2)$ matrix functions. In the underlying paper, based on Refs. $[2,6,26,30]$, we will first define a proper inner product on the space of square integral circulant $(2 \times 2)$ matrix functions defined on the boundary of a bounded sub-domain in even dimensional Euclidean space, and introduce the matrix Szegö projection operator to be orthogonal for the Hardy space of Hermitean monogenic functions defined on a bounded sub-domain of even dimensional Euclidean space. Then we will establish the Kerzman-Stein formula, which is closely related to the matrix Szegö projection operator and the Hardy projection operator onto the Hardy space of Hermitean monogenic functions defined on a bounded sub-domain, and present the matrix Szegö projection operator in terms of the Hardy projection operator and its adjoint, 
explicitly. Lastly, we will give the explicit matrix Szegö kernel function for the Hardy space on the sphere. As an application, we get the solution to a boundary value problem for matrix functions.

The paper is organized as follows. In Section 2, we recall some basic facts about Hermitean Clifford analysis which will be needed in the sequel. In Section 3, we will introduce the matrix Szegö projection operator for the Hardy space of Hermitean monogenic functions defined on a bounded sub-domain, establish the Kerzman-Stein formula which closely connects the matrix Szegö projection operator, and present the matrix Szegö projection operator in terms of the Hardy projection operator and its adjoint in the Hermitean Clifford setting. At last we will construct the explicit matrix Szegö kernel function for the Hardy space, and get the solution to Hermitean Dirichlet problem.

\section{Preliminaries}

In this section we recall some basic facts about Clifford algebra and Hermitean Clifford analysis which will be needed in the sequel. More details can be also seen in Refs. e.g. [20-24].

Let $\left(e_{1}, \ldots, e_{m}\right)$ be an orthogonal basis of Euclid space $\mathbb{R}^{m}$, the complex Clifford algebra $\mathbb{C}_{m}$, which is constructed over $\mathbb{R}^{m}$, its geometric multiplication is governed by the rules $e_{j} e_{k}+$ $e_{k} e_{j}=-2 \delta_{j k}, j, k=1, \ldots, m$. The $\mathbb{C}_{m}$ thus is generated additively by elements of the form $e_{A}=e_{j_{1}} \cdots e_{j_{k}}$, where $A=\left\{j_{1}, \ldots, j_{k}\right\} \subset\{1, \ldots, m\}, j_{1}<\cdots<j_{k}$, while for $A=\emptyset$, one puts $e_{\emptyset}=1$, the identity element. Any Clifford number $a$ in $\mathbb{C}_{m}$ may thus be written as $a=\sum_{A} a_{A} e_{\mathcal{A}}, a_{A} \in \mathbb{C}$, and its Hermitean conjugate $a^{\dagger}$ is defined by $a^{\dagger}=\sum_{A} a_{A}^{c} \bar{e}_{A}$, where the bar denotes the usual real Clifford algebra conjugation and ${ }^{c}$ denotes the standard complex conjugation. The Euclidean space $\mathbb{R}^{m}$ is embedded in $\mathbb{C}_{m}$ by identifying $\left(x_{1}, x_{2}, \ldots, x_{m}\right)$ with the Clifford vector $\underline{X}$ given by $\underline{X}=\sum_{j=1}^{m} e_{j} x_{j}$. Note that the square of a vector $\underline{X}$ is scalar valued and equals the norm squared up to a minus sign $\underline{X}^{2}=-\langle\underline{X}, \underline{X}\rangle=-|\underline{X}|^{2}$. The Fischer dual of the vector $\underline{X}$ is the vector valued first order differential operator $\partial_{\underline{X}}=\sum_{j=1}^{m} e_{j} \partial_{x_{j}}$ is called Dirac operator. It is precisely this Dirac operator which underlies the notion of monogenicity of a function, a notion which is the higher dimensional counterpart or holomorphy in the complex plane. As the Dirac operator factorizes the Laplacian, $\Delta_{m}=-\partial_{\underline{X}}^{2}$, monogenicity can be regarded as a refinement of harmonicity.

Hereby, introducing Hermitean Clifford analysis is based on the so-called almost complex structure on it, i.e., an $\mathrm{SO}(m)$-element $J$, satisfying $J^{2}=-\mathbf{1}_{m}$. This forces the dimension $m$ to be even, whence from now on, we will put $m=2 n$. In terms of our basis, a particular realization of the almost complex structure is given by $J\left(e_{2 j-1}\right)=-e_{2 j}$ and $J\left(e_{2 j}\right)=e_{2 j-1}, j=1, \ldots, n$.

The real Clifford vector and the Dirac operator are denoted by

$$
\underline{X}=\sum_{j=1}^{n}\left(e_{2 j-1} x_{2 j-1}+e_{2 j} x_{2 j}\right), \quad \partial_{\underline{X}}=\sum_{j=1}^{n}\left(e_{2 j-1} \partial_{x_{2 j-1}}+e_{2 j} \partial_{x_{2 j}}\right),
$$

as well as their counterparts

$$
\underline{X} \mid=\sum_{j=1}^{n}\left(e_{2 j-1} x_{2 j}-e_{2 j} x_{2 j-1}\right), \quad \partial_{\underline{X} \mid}=\sum_{j=1}^{n}\left(e_{2 j-1} \partial_{x_{2 j}}-e_{2 j} \partial_{x_{2 j-1}}\right) .
$$


The Hermitean Clifford variables $\underline{Z}$ and $\underline{Z}^{\dagger}$ then given by

which satisfy

$$
\underline{Z}=\frac{1}{2}(\underline{X}+i \underline{X} \mid), \quad \underline{Z}^{\dagger}=-\frac{1}{2}(\underline{X}-i \underline{X} \mid),
$$

$$
J(\underline{Z})=-i \underline{Z}, J\left(\underline{Z}^{\dagger}\right)=i \underline{Z}^{\dagger} .
$$

Hermitean Clifford analysis then focuses on simultaneous null solutions of two Hermitean Dirac operators $\partial_{\underline{Z}}$ and $\partial_{\underline{Z}^{\dagger}}$, introduced by

$$
\partial_{\underline{Z}^{\dagger}}=\frac{1}{4}\left(\partial_{\underline{X}}+i \partial_{\underline{X} \mid}\right), \quad \partial_{\underline{Z}}=-\frac{1}{4}\left(\partial_{\underline{X}}-i \partial_{\underline{X}}\right) .
$$

From observation we have $(\underline{Z})^{2}=\left(\underline{Z}^{\dagger}\right)^{2}=0$ and $\left(\partial_{\underline{Z}}\right)^{2}=\left(\partial_{\underline{Z}^{\dagger}}\right)^{2}=0$, and $\Delta_{2 n}=4\left(\partial_{\underline{Z}} \partial_{\underline{Z}^{\dagger}}+\right.$ $\left.\partial_{\underline{Z}^{\dagger}} \partial_{\underline{Z}}\right), \underline{Z} \underline{Z}^{\dagger}+\underline{Z}^{\dagger} \underline{Z}=|\underline{Z}|^{2}=\left|\underline{Z}^{\dagger}\right|^{2}=|\underline{X}|^{2}=\left.|\underline{X}|\right|^{2}$.

The fundamental solutions of the Dirac operators $\partial_{\underline{X}}, \partial_{\underline{X}}$ are

$$
E(\underline{X})=\frac{2}{\omega_{2 n}} \frac{\bar{X}}{|\underline{X}|^{2 n}}, \quad E(\underline{X} \mid)=\frac{2}{\omega_{2 n}} \frac{\bar{X} \mid}{|\underline{X}|^{2 n}}, \quad \underline{X} \in \mathbb{R}^{2 n} \backslash\{0\},
$$

where $\omega_{2 n}$ denotes the surface area of the unit sphere in $\mathbb{R}^{2 n}$. We introduce

$$
\mathcal{E}(\underline{Z})=-(E+i E \mid)=\frac{2}{\omega_{2 n}} \frac{\underline{Z}}{|\underline{Z}|^{2 n}}, \quad \mathcal{E}^{\dagger}(\underline{Z})=(E-i E \mid)=\frac{2}{\omega_{2 n}} \frac{\underline{Z}^{\dagger}}{|\underline{Z}|^{2 n}} .
$$

For further use, we introduce the oriented surface elements $d \sigma_{\underline{Z}}$ and $d \sigma_{\underline{Z}^{\dagger}}$ by

$$
d \sigma_{\underline{Z}}=-\frac{1}{4}(-1)^{\frac{n(n+1)}{2}}(2 i)^{n}\left(d \sigma_{\underline{X}}-i d \sigma_{\underline{X} \mid}\right), \quad d \sigma_{\underline{Z}^{\dagger}}=-\frac{1}{4}(-1)^{\frac{n(n+1)}{2}}(2 i)^{n}\left(d \sigma_{\underline{X}}+i d \sigma_{\underline{X}}\right),
$$

where $d \sigma_{\underline{X}}=\sum_{j=1}^{2 n} e_{j}(-1)^{j-1} \widehat{d x_{j}}$ and $d \sigma_{\underline{X} \mid}=J\left[d \sigma_{\underline{X}}\right]$ with

$$
\widehat{d x_{j}}=d x_{1} \wedge \cdots \wedge d x_{j-1} \wedge d x_{j+1} \wedge \cdots \wedge d x_{2 n} .
$$

We denote the outward pointing unit normal vector at $X \in \partial \Omega$ by $\nu(\underline{X})$ and $d S(\underline{X})$ for the element on $\partial \Omega$, leading to $d \sigma_{\underline{X}}=\nu(\underline{X}) d S(\underline{X}), d \sigma_{\underline{X} \mid}=\nu \mid(\underline{X}) d S(\underline{X})$.

In this context the functions under consideration are defined on an open subset $\Omega$ of $\mathbb{R}^{2 n}$ and take values in the Clifford algebra $\mathbb{C}_{2 n}$. They are of the form $f=\sum_{\mathcal{A}} f_{\mathcal{A}} e_{\mathcal{A}}$, where the functions $f_{\mathcal{A}}$ are complex-valued. Whenever a property such as continuity, differentiability, $L_{p^{-}}$ integrable and so forth is ascribed to $f$, it is meant that all the components $f_{\mathcal{A}}$ possess the cited property. Let $g_{1}, g_{2}$ be $\mathbb{C}_{2 n}$-valued functions defined in $\Omega \subset \mathbb{R}^{2 n}$, we consider the corresponding circulant $(2 \times 2)$ matrix function in the following

$$
\mathbf{G}_{2}^{1}=\left(\begin{array}{ll}
g_{1} & g_{2} \\
g_{2} & g_{1}
\end{array}\right) .
$$

In what follows, the operations of matrices such as addition and multiplication, and the operations between the complex numbers and the matrices, respectively, keep to the operation rules of the usual numerical matrices and of multiplication between the complex numbers and the usual numerical matrices. Let $\Omega \subset \mathbb{R}^{2 n}$ be a bounded sub-domain with smooth boundary $\partial \Omega$. Functions taking values in $\mathbb{C}_{2 n}$ defined on $\Omega \cup \partial \Omega$ will be considered. Notions of continuity, differentiability and integrability of $\mathbf{G}_{2}^{1}$ are introduced entry-wise. For instance, the circulant $(2 \times 2)$ matrix function $\mathbf{G}_{2}^{1} \in \mathbf{C}^{k}\left(\Omega, \mathbb{C}_{2 n}\right), \mathbf{H}^{\mu}\left(\Omega, \mathbb{C}_{2 n}\right), \mathbf{L}_{p}\left(\Omega, \mathbb{C}_{2 n}\right)$ and so on which mean 
each entry of $\mathbf{G}_{2}^{1}$ belongs to $C^{k}\left(\Omega, \mathbb{C}_{2 n}\right), H^{\mu}\left(\Omega, \mathbb{C}_{2 n}\right), L_{p}\left(\Omega, \mathbb{C}_{2 n}\right)$ and so on. We introduce the particular circulant $(2 \times 2)$ matrices

$$
\mathbf{D}_{\left(\underline{Z}, \underline{Z}^{\dagger}\right)}=\left(\begin{array}{cc}
\partial_{\underline{Z}} & \partial_{\underline{Z}^{\dagger}} \\
\partial_{\underline{Z}^{\dagger}} & \partial_{\underline{Z}}
\end{array}\right), \quad \mathcal{E}=\left(\begin{array}{cc}
\mathcal{E} & \mathcal{E}^{\dagger} \\
\mathcal{E}^{\dagger} & \mathcal{E}
\end{array}\right), \quad \underline{\delta}=\left(\begin{array}{cc}
\delta & 0 \\
0 & \delta
\end{array}\right),
$$

where $\delta$ is the Dirac delta distribution in $\mathbb{R}^{2 n}$, then $\mathbf{D}_{\left(\underline{Z}, \underline{Z}^{\dagger}\right)} \mathcal{E}=\underline{\delta}(\underline{Z})$, i.e. $\mathcal{E}$ is the fundamental solution of $\mathbf{D}_{\left(\underline{Z}, \underline{Z}^{\dagger}\right)}$ (see Refs. e.g. [20-24, 26]).

Definition 2.1 $\mathbf{G}_{2}^{1} \in \mathbf{C}^{1}\left(\Omega, \mathbb{C}_{2 n}\right)$ is called as (left) $\mathbf{H}$-monogenic in $\Omega$ if and only if it satisfies the system $\mathbf{D}_{\left(\underline{Z}, \underline{Z}^{\dagger}\right)} \mathbf{G}_{2}^{1}=\mathbf{0}$, where $\mathbf{0}$ denotes the $(2 \times 2)$ matrix with zero entries.

For $g_{i} \in L_{p}\left(\partial \Omega, \mathbb{C}_{2 n}\right), 1<p<+\infty, i=1,2$, we define the orthogonal Cauchy type integrals as

$$
\mathcal{C}\left[g_{i}\right](\underline{Y})=\int_{\partial \Omega} E(\underline{X}-\underline{Y}) d \sigma_{\underline{X}} g_{i}(\underline{X}), \quad \mathcal{C}\left|\left[g_{i}\right](\underline{Y})=\int_{\partial \Omega} E\right|(\underline{X}-\underline{Y}) d \sigma_{\underline{X}} g_{i}(\underline{X}), \underline{Y} \notin \partial \Omega,
$$

which are well-defined (see Refs. e.g. $[12,13]$ ), where $E(\underline{X}), E \mid(\underline{X})$ and $d \sigma_{\underline{X}}, d \sigma_{\underline{X}}$ as above. Then for

$$
\partial_{\underline{Y}} \mathcal{C}\left[g_{i}\right](\underline{Y})=0, \quad \partial_{\underline{Y} \mid} \mathcal{C} \mid\left[g_{i}\right](\underline{Y})=0, \quad \underline{Y} \notin \partial \Omega, i=1,2 .
$$

For $\mathbf{G}_{2}^{1} \in \mathbf{L}_{p}\left(\partial \Omega, \mathbb{C}_{2 n}\right)$, the Hermitean Cauchy type integral is defined by

$$
\left[\mathbf{C G}_{2}^{1}\right](\underline{Y})=\int_{\partial \Omega} \mathcal{E}(\underline{Z}-\underline{V}) d \boldsymbol{\Sigma}_{\left(\underline{Z}, \underline{Z}^{\dagger}\right)} \mathbf{G}_{2}^{1}(\underline{X}), \quad \underline{Y} \notin \partial \Omega,
$$

where

$$
d \boldsymbol{\Sigma}_{\left(\underline{Z}, \underline{Z}^{\dagger}\right)}=\left(\begin{array}{cc}
d \sigma_{\underline{Z}} & -d \sigma_{\underline{Z}^{\dagger}} \\
-d \sigma_{\underline{Z}^{\dagger}} & d \sigma_{\underline{Z}}
\end{array}\right)
$$

\section{Szegö projection operator}

In this section we will introduce the matrix Szegö projection operator for the Hardy space of Hermitean monogenic functions defined on a bounded sub-domain, establish the Kerzman-Stein formula, and present the matrix Szegö projection operator in terms of the Hardy projection operator and its adjoint, explicitly.

Inspired by the inner product $\langle\cdot, \cdot\rangle_{L_{2}}$ on $L_{2}\left(\partial \Omega, \mathbb{C}_{2 n}\right)$, given by

$$
\left\langle g_{1}, g_{2}\right\rangle_{L_{2}}=\left[\int_{\partial \Omega} g_{1}^{\dagger}(\underline{X}) g_{2}(\underline{X}) d S_{\underline{X}}\right]_{0}, \quad \forall g_{1}, g_{2} \in L_{2}\left(\partial \Omega, \mathbb{C}_{2 n}\right),
$$

where $[\cdot]_{0}$ denotes the scale part of any $\cdot$ in $\mathbb{C}_{2 n}$. We introduce the following bi-linear form on the vector space $\mathbf{L}_{2}\left(\partial \Omega, \mathbb{C}_{2 n}\right)$

$$
\begin{aligned}
\langle\cdot, \cdot\rangle_{\mathbf{L}_{2}}: \mathbf{L}_{2}(\partial \Omega) \times \mathbf{L}_{2}(\partial \Omega) & \rightarrow \mathbb{C} \\
\left\langle\mathbf{F}_{2}^{1}, \mathbf{G}_{2}^{1}\right\rangle_{\mathbf{L}_{2}} & \mapsto\left\langle f_{1}, g_{1}\right\rangle_{L_{2}}+\left\langle f_{2}, g_{2}\right\rangle_{L_{2}},
\end{aligned}
$$

where $\mathbf{F}_{2}^{1}$ is defined similarly to $\mathbf{G}_{2}^{1}$ as Section 2 .

Then, by directly calculating, for arbitrary $\mathbf{F}_{2}^{1}, \mathbf{G}_{2}^{1}, \mathbf{H}_{2}^{1} \in \mathbf{L}_{2}\left(\partial \Omega, \mathbb{C}_{2 n}\right)$ and $\lambda \in \mathbb{C}$, we can check that

(i) $\left\langle\mathbf{F}_{2}^{1}, \lambda \mathbf{G}_{2}^{1}+\mathbf{H}_{2}^{1}\right\rangle_{\mathbf{L}_{2}}=\lambda\left\langle\mathbf{F}_{2}^{1}, \mathbf{G}_{2}^{1}\right\rangle_{\mathbf{L}_{2}}+\left\langle\mathbf{F}_{2}^{1}, \mathbf{H}_{2}^{1}\right\rangle_{\mathbf{L}_{2}}$,

(ii) $\left(\left\langle\mathbf{F}_{2}^{1}, \mathbf{G}_{2}^{1}\right\rangle_{\mathbf{L}_{2}}\right)^{\dagger}=\left\langle\mathbf{G}_{2}^{1}, \mathbf{F}_{2}^{1}\right\rangle_{\mathbf{L}_{2}}$, 
(iii) $\left\langle\mathbf{G}_{2}^{1}, \mathbf{G}_{2}^{1}\right\rangle_{\mathbf{L}_{2}} \geq 0$ and $\left\langle\mathbf{G}_{2}^{1}, \mathbf{G}_{2}^{1}\right\rangle_{\mathbf{L}_{2}}=0$ if and only if $\mathbf{G}_{2}^{1}=\mathbf{0}$.

Thus, $\langle\cdot, \cdot\rangle_{\mathbf{L}_{2}}$ is a inner product, which derives the norm on $\mathbf{L}_{2}\left(\partial \Omega, \mathbb{C}_{2 n}\right)$ by

$$
\left\|\mathbf{G}_{2}^{1}\right\|=\sqrt{\left\langle g_{1}, g_{1}\right\rangle_{L_{2}}+\left\langle g_{2}, g_{2}\right\rangle_{L_{2}}} .
$$

Hence, $\left(\mathbf{L}_{2}(\partial \Omega),\|\cdot\|\right)$ is the Hilbert space, which is different from the space of $\mathbf{L}_{2}(\partial \Omega)$ in Refs. e.g. $[26,30]$. Under this setting, we have the following lemma without proof, which was also stated in $[24,26,30]$ in the sense of different topology. For convenience without confusion and ambiguity, $\left(\mathbf{L}_{2}(\partial \Omega),\|\cdot\|\right)$ still denotes by $\mathbf{L}_{2}(\partial \Omega)$.

Lemma 3.1 Let $\Omega$ be a non-empty, open and bounded subset of $\mathbb{R}^{2 n}$ with smooth boundary $\partial \Omega$. $\quad\left[\mathbf{C G}_{2}^{1}\right](\underline{X})$ is defined similarly to $\left[\mathbf{C G}_{2}^{1}\right](\underline{Y})$ as Section 2. If $\mathbf{G}_{2}^{1}(\underline{X}) \in \mathbf{L}_{p}\left(\partial \Omega, \mathbb{C}_{2 n}\right)$, $(1<p<+\infty)$, then, for arbitrary $\underline{T} \in \partial \Omega$,

(i) $\forall \underline{X} \in \mathbb{R}^{2 n} \backslash \partial \Omega, \mathbf{D}_{\left(\underline{Z}, \underline{Z}^{\dagger}\right)} \mathbf{G}_{2}^{1}(\underline{X})=\mathbf{0}$, i.e. $\mathbf{G}_{2}^{1}(\underline{X})$ is $\mathbf{H}$-monogenic in $\mathbb{R}^{2 n} \backslash \partial \Omega$,

(ii) $\left[\mathbf{C G}_{2}^{1}\right]^{ \pm}(\underline{T}) \triangleq \lim _{\Omega^{ \pm} \ni \underline{X} \rightarrow \underline{T}}\left[\mathbf{C G}_{2}^{1}\right](\underline{X})=(-1)^{\frac{n(n+1)}{2}} \frac{(2 i)^{n}}{2}\left( \pm \mathbf{G}_{2}^{1}(\underline{T})+\left[\mathbf{H G}_{2}^{1}\right](\underline{T})\right)$;

(iii) $\left[\mathbf{C G}_{2}^{1}\right]^{ \pm}(\underline{T}) \in \mathbf{L}_{p}\left(\partial \Omega, \mathbb{C}_{2 n}\right)$,

where the limits of (ii) mean the non-tangential limits, which is the same in the following context,

$$
\mathbf{H}=\frac{1}{2}\left(\begin{array}{rr}
\mathcal{H}+\mathcal{H} \mid & -\mathcal{H}+\mathcal{H} \mid \\
-\mathcal{H}+\mathcal{H} \mid & \mathcal{H}+\mathcal{H} \mid
\end{array}\right)
$$

and

$$
\begin{aligned}
& {[\mathcal{H} f](\underline{T})=\text { p.v.2 } \int_{\partial \Omega} E(\underline{Y}-\underline{T}) d \sigma_{\underline{Y}} f(\underline{Y}), \underline{T} \in \partial \Omega,} \\
& {[\mathcal{H} \mid f](\underline{T})=\text { p.v. } 2 \int_{\partial \Omega} E \mid(\underline{Y}-\underline{T}) d \sigma_{\underline{Y} \mid} f(\underline{Y}), \underline{T} \in \partial \Omega,}
\end{aligned}
$$

which are both Cauchy principle value integrals in the sense of $L_{p}(1<p<+\infty)$. When the variables are omitted without confusion and ambiguity, for convenience $[\mathcal{H} f](\underline{T}),[\mathcal{H} \mid f](\underline{T})$ are for short of $\mathcal{H} f, \mathcal{H} \mid f$, respectively, and it is also similar in the following context.

we will consider the Hardy space

$\mathbb{H}^{2}(\Omega)=\left\{\mathbf{G}_{2}^{1} \mid \mathbf{G}_{2}^{1}\right.$ is $\mathbf{H}$-monogenic in $\Omega$, and has non-tangential $\mathbf{L}_{2}(\partial \Omega)$-boundary values $\}$, and $\mathbb{H}^{2}(\partial \Omega)$ denotes the $\mathbf{L}_{2}(\partial \Omega)$-closure of the set of boundary values of elements of $\mathbb{H}^{2}(\Omega)$.

Remark 3.2 Associating the Hardy space with (ii) of Lemma 3.1, the Hermitean Cauchy transform $\mathbf{C}$ maps $\mathbf{L}_{2}\left(\partial \Omega, \mathbb{C}_{2 n}\right)$ onto $\mathbb{H}^{2}(\partial \Omega)$ for arbitrary $\mathbf{G}_{2}^{1} \in \mathbf{L}_{2}\left(\partial \Omega, \mathbb{C}_{2 n}\right)$, which is skew and so-called the Hardy projection.

Associating the definition of the above $\mathbb{C}$-valued inner product on $\mathbf{L}_{2}(\partial \Omega)$, we have the following lemma which is only stated without proof.

Lemma 3.3 Suppose that $\mathbf{H}, \mathbf{L}_{2}(\partial \Omega)$ and $\mathbb{H}^{2}(\partial \Omega)$ as Lemma 3.1 and Remark 3.2. Then

(i) $\mathbf{H}^{2}=\mathbf{I}$,

(ii) $\mathbf{H}^{*}=\underline{\nu} \mathbf{H} \underline{\nu}$,

(iii) for arbitrary $\mathbf{G}_{2}^{1} \in \mathbf{L}_{2}(\partial \Omega), \mathbf{H G}_{2}^{1}=\mathbf{G}_{2}^{1}$ if and only if $\mathbf{G}_{2}^{1} \in \mathbb{H}^{2}(\partial \Omega)$,

(iv) $\mathbf{L}_{2}(\partial \Omega)=\mathbb{H}^{2}(\partial \Omega) \oplus \underline{\nu} \mathbb{H}^{2}(\partial \Omega)$, (w.r.t. $\left.\langle\cdot, \cdot\rangle_{\mathbf{L}_{2}}\right)$,

where $\mathbf{I}$ denotes $(2 \times 2)$ identity matrix operator, $\mathbf{H}^{*}$ means the adjoint operators of $\mathbf{H}$ on 
$\mathbf{L}_{2}(\partial \Omega)$ and

$$
\underline{\nu}=\frac{1}{2}\left(\begin{array}{rr}
\nu+\nu \mid & -\nu+\nu \mid \\
-\nu+\nu \mid & \nu+\nu \mid
\end{array}\right),
$$

with $\nu$ being the outward pointing unit normal vector at $\underline{X} \in \partial \Omega$ and $J(\nu)=\nu \mid$.

Remark 3.4 The results similar to Lemma 3.3 were also mentioned in Refs. [24, 26] with respect to $\left(\mathbb{C}_{2 n}\right)^{2 \times 2}$-valued inner product, which does not derive a norm and is different from our $\mathbb{C}$-valued inner product on $\mathbf{L}_{2}(\partial \Omega)$.

Starting with (ii) of Lemma 3.3, the matrix orthogonal projection operator $\mathbf{S}$ from $\mathbf{L}_{2}(\partial \Omega)$ onto $\mathbb{H}^{2}(\partial \Omega)$, which is so-called the matrix Szegö projection operator, may be Hermitean monogenically extended to $\mathbb{H}^{2}(\Omega)$ by

$$
\mathbf{S}\left[\mathbf{G}_{2}^{1}(\underline{X})\right]=\int_{\partial \Omega} \underline{\mathbf{S}}_{\underline{X}}(\underline{Y}) \mathbf{G}_{2}^{1}(\underline{Y}) d S_{\underline{Y}},
$$

where $\underline{\mathbf{S}}_{\underline{X}}(\underline{Y})$ is so-called the matrix Szegö kernel. That is,

$$
\mathbf{S}\left[\mathbf{G}_{2}^{1}(\underline{X})\right]=\mathbf{G}_{2}^{1}(\underline{X}) \text {, for arbitrary } \underline{X} \in \Omega .
$$

Remark 3.5 Particularly, when $\Omega=B(1)$ the unit ball centered at 0 of $\mathbb{R}^{2 n}, \partial \Omega=S^{2 n}$ the unit sphere of $\mathbb{R}^{2 n}$ and $\nu(\underline{W})=\underline{W}, \nu|(\underline{W})=\underline{W}|$ for arbitrary $\underline{W} \in S^{2 n}$. Then

$$
\mathbf{L}_{2}\left(S^{2 n}\right)=\left.\mathbb{H}^{2}\left(S^{2 n}\right) \oplus \underline{\nu}\right|_{S^{2 n}} \mathbb{H}^{2}\left(S^{2 n}\right),
$$

where

$$
\left.\underline{\nu}\right|_{S^{2 n}}=\frac{1}{2}\left(\begin{array}{rr}
\underline{W}+\underline{W} \mid & -\underline{W}+\underline{W} \mid \\
-\underline{W}+\underline{W} \mid & \underline{W}+\underline{W} \mid
\end{array}\right) .
$$

We consider the Dirichlet problem as follows, given the boundary data $\mathbf{G}_{2}^{1} \in \mathbf{L}_{2}\left(S^{2 n}, \mathbb{C}_{2 n}\right)$, find the function $\mathbf{F}_{2}^{1}$ such that

$$
\left\{\begin{array}{l}
\underline{\Delta} \mathbf{F}_{2}^{1}(\underline{X})=\mathbf{0}, \underline{X} \in B(1), \\
\mathbf{F}_{2}^{1}(\underline{W})=\mathbf{G}_{2}^{1}(\underline{W}), \underline{W} \in S^{2 n},
\end{array}\right.
$$

where

$$
\underline{\Delta}=\left(\begin{array}{ll}
\Delta_{2 n} & 0 \\
0 & \Delta_{2 n}
\end{array}\right) .
$$

it is easy to verify that (3.3) is equivalent to the system

$$
\left\{\begin{array}{l}
\Delta_{2 n} f_{1}(\underline{X})=0, \underline{X} \in B(1), \\
f_{1}(\underline{W})=g_{1}(\underline{W}), \underline{W} \in S^{2 n}, \\
\Delta_{2 n} f_{2}(\underline{X})=0, \underline{X} \in B(1), \\
f_{2}(\underline{W})=g_{2}(\underline{W}), \underline{W} \in S^{2 n},
\end{array}\right.
$$

In virtue of $(i v)$ in Lemma 3.3, we have

$$
\mathbf{G}_{2}^{1}=\mathbf{H}_{2}^{1}+\underline{\nu} \mathbf{K}_{2}^{1}
$$

where $\mathbf{H}_{2}^{1}, \mathbf{K}_{2}^{1} \in \mathbb{H}^{2}\left(S^{2 n}\right)$ are defined similarly to $\mathbf{G}_{2}^{1}$. 
Then the above Dirichlet problem (3.3) exists the unique solution. Moreover the solution is formulated in the following form

$$
\mathbf{G}_{2}^{1}(\underline{X})=\widetilde{\mathbf{H}}_{2}^{1}+\underline{\mathcal{X}} \widetilde{\mathbf{K}}_{2}^{1}, \quad \underline{X} \in B(1),
$$

where $\widetilde{\mathbf{H}}_{2}^{1}, \widetilde{\mathbf{K}}_{2}^{1} \in \mathbb{H}^{2}(B(1))$ are Hermitean monogenic extension of $\mathbf{H}_{2}^{1}, \mathbf{K}_{2}^{1}$, respectively, i.e., $\mathbf{H}_{2}^{1}, \mathbf{K}_{2}^{1}$ are the non-tangential boundary value limits of $\widetilde{\mathbf{H}}_{2}^{1}, \widetilde{\mathbf{K}}_{2}^{1}$ and

$$
\underline{\mathcal{X}}=\left(\begin{array}{rr}
\underline{X}+\underline{X} \mid & -\underline{X}+\underline{X} \mid \\
-\underline{X}+\underline{X} \mid & \underline{X}+\underline{X} \mid
\end{array}\right) .
$$

In what follows, we introduce the matrix Kerzman-Stein operator on $\mathscr{L}_{2}(\partial \Omega)$ by

$$
\underline{\mathcal{A}}=\frac{1}{2}\left(\begin{array}{rr}
\mathcal{A}+\mathcal{A} \mid & -\mathcal{A}+\mathcal{A} \mid \\
-\mathcal{A}+\mathcal{A} \mid & \mathcal{A}+\mathcal{A} \mid
\end{array}\right),
$$

where

$$
\mathcal{A}=\mathcal{C}-\mathcal{C}^{*}, \mathcal{A}|=\mathcal{C}|-\left.\mathcal{C}\right|^{*}
$$

are both well-defined, $\mathcal{C}^{*}$ and $\left.\mathcal{C}\right|^{*}$ denote the adjoint operators of $\mathcal{C}$ and $\mathcal{C} \mid$ on the Hilbert space of $\mathbf{L}_{2}\left(\partial \Omega, \mathbb{C}_{2 n}\right)$, respectively, given by

$$
\begin{aligned}
\mathcal{C}^{*} & =\frac{1}{2}(1+\nu \mathcal{H} \nu): L_{2}\left(\partial \Omega, \mathbb{C}_{2 n}\right) \rightarrow H^{2}(\partial \Omega), \\
\left.\mathcal{C}\right|^{*} & =\frac{1}{2}(1+\nu|\mathcal{H}| \nu \mid): L_{2}\left(\partial \Omega, \mathbb{C}_{2 n}\right) \rightarrow H^{2}(\partial \Omega),
\end{aligned}
$$

with $H^{2}(\partial \Omega)$ being $L_{2}\left(\partial \Omega, \mathbb{C}_{2 n}\right)$-closure of the set of boundary values of elements of

$H^{2}(\Omega)=\left\{g \mid \partial_{\underline{X}} g=0\right.$ in $\Omega$ and having non-tangential $L_{2}(\partial \Omega)$-boundary values $\}$

$\nu, \nu|, \mathcal{H}, \mathcal{H}|$ as Section 2, and 1 being the identity operator. More detail can be seen in Refs. $[10,12,11]$.

Applying Lemma 3.3, we directly get the following lemma.

Lemma 3.6 Let $\mathcal{A}$ and $\mathcal{A} \mid$ be as term (3.6), and $\mathbf{C}$ be as Remark 3.2. Then

$$
\underline{\mathcal{A}}=\mathbf{C}-\mathbf{C}^{*}=\mathbf{H}-\mathbf{H}^{*}, \text { i.e., } \underline{\mathcal{A}}=\mathbf{H}-\underline{\nu} \mathbf{H} \underline{\nu}
$$

where $\mathbf{H}^{*}$, seen in Lemma 3.3 , and $\mathbf{C}^{*}=\frac{1}{2}\left(\mathbf{I}+\mathbf{H}^{*}\right)$ mean the adjoint operators of $\mathbf{H}$ and $\mathbf{C}$ on $\mathbf{L}_{2}(\partial \Omega)$.

Theorem 3.7 Let $\mathbf{S}$ be as term (3.2), and $\mathbf{C}$ be as Remark 3.2. Then

$$
\mathbf{S}(\mathbf{I}+\underline{\mathcal{A}})=\mathbf{C}
$$

where $\mathbf{I}$ denotes $(2 \times 2)$ identity matrix operator.

Proof Since the matrix operator $\mathbf{S}$ is orthogonal projection operator on the Hilbert space $\mathbf{L}_{2}(\partial \Omega), \mathbf{S}=\mathbf{S}^{*}$. Noticing that the operators $\mathbf{S}$ and $\mathbf{C}$ are orthogonal and skew projection operators from $\mathbf{L}_{2}(\partial \Omega)$ to $\mathbb{H}^{2}(\partial \Omega)$, respectively, then $\mathbf{S C}$ and $\mathbf{C S}$ are both operators from $\mathbf{L}_{2}(\partial \Omega)$ to $\mathbb{H}^{2}(\partial \Omega)$. In particular, operators $\mathbf{S}$ and $\mathbf{C}$ are both the identical operators on $\mathbb{H}^{2}(\partial \Omega)$, respectively. Therefore, we have

$$
\mathbf{S C}=\mathbf{C} \text { and } \mathbf{C S}=\mathbf{S} \text {. }
$$


Applying the property of the adjoint operator on the Hilbert space of $\mathbf{L}_{2}(\partial \Omega)$ (see Refs. e.g. $[3,10,12]$ or monographs of functional analysis $),(\mathbf{S C})^{*}$ is well-defined and $(\mathbf{S C})^{*}=\mathbf{C}^{*} \mathbf{S}^{*}$, where $\mathbf{C}^{*}$ means the adjoint operator of $\mathbf{C}$ acting on $\mathbf{L}_{2}(\partial \Omega)$. Taking the adjoint operators with respect to $\langle., .\rangle_{\mathbf{L}_{2}}$, we have

$$
\mathbf{C}^{*} \mathbf{S}=(\mathbf{S C})^{*}=\mathbf{C}^{*} \text { and } \mathbf{S C}^{*}=(\mathbf{C S})^{*}=\mathbf{S} .
$$

Hence, associating terms (3.9) with (3.10), we get

$$
\mathbf{S C}-\mathbf{S C}^{*}=\mathbf{C}-\mathbf{S}
$$

Therefore, one has

$$
\mathbf{S}(\mathbf{I}+\underline{\mathcal{A}})=\mathbf{C} .
$$

Thus, the proof of the result is complete.

Remark 3.8 Theorem 3.7 characterizes the relationship between Hermitean Hardy projection operator and matrix Szegö projection operator, which is the generalization of the wellknown Kerzman-Stein formula into the setting of Hermitean Clifford analysis.

We define the matrix operator as follows

$$
\underline{\mathcal{B}}=\frac{1}{2}\left(\begin{array}{cc}
(1+\mathcal{A})^{-1}+(1+\mathcal{A} \mid)^{-1} & -(1+\mathcal{A})^{-1}+(1+\mathcal{A} \mid)^{-1} \\
-(1+\mathcal{A})^{-1}+(1+\mathcal{A} \mid)^{-1} & (1+\mathcal{A})^{-1}+(1+\mathcal{A} \mid)^{-1}
\end{array}\right)
$$

where 1 denotes the identity operator on $L_{2}\left(\partial \Omega, \mathbb{C}_{2 n}\right)$.

Observing from term (3.6), the operators $1+\mathcal{A}$ and $1+\mathcal{A} \mid$ are invertible on $L_{2}\left(\partial \Omega, \mathbb{C}_{2 n}\right)$, which could be also seen in Lemma 4.5 in [6] or [3], the matrix operator $\underline{\mathcal{B}}$ is well defined on $\mathbf{L}_{2}(\partial \Omega)$.

Theorem 3.9 Let $\mathbf{S}$ and $\mathbf{C}$ be as Theorem 3.7. Then the matrix Szegö projection operator is explicitly formulated by

$$
\mathbf{S}=\mathbf{C}(\mathbf{I}+\underline{\mathcal{A}})^{-1}
$$

where $\mathbf{I}$ denotes $(2 \times 2)$ identity matrix operator.

Proof Applying term (3.7), we know that operator $\underline{\mathcal{A}}$ is anti-self conjugate. This implies that the spectra of operator $\underline{\mathcal{A}}$ are pure imaginary numbers. Hence, operator $\mathbf{I}+\underline{\mathcal{A}}$ is invertible. Moreover, by calculating directly, we get $(\mathbf{I}+\underline{\mathcal{A}})^{-1}=\underline{\mathcal{B}}$. It follows the result.

Remark 3.10 Equation (3.11) is our basic desired formula, which gives a characterization of the matrix Szegö projection operator. Notice that, for smooth boundary of a bounded domain, the principal value parts in $\mathbf{C}-\mathbf{C}^{*}$ have disappeared, which leads to the inverse of operator $\underline{\mathcal{A}}$. For smooth boundary of general unbounded domains, it is more complicated, which we do not focus on.

\section{$4 \quad$ Szegö kernel}

In this section we construct the explicit matrix Szegö kernel for the Hardy space $\mathbb{H}^{2}\left(S^{2 n}\right)$. As an application of it, we get the solution to a boundary value problem for matrix functions in terms of integral formula. 
We introduce the functions

$$
K(\underline{X}, \underline{Y})=-\frac{1}{\omega_{2 n}} \frac{1+\underline{X Y}}{|1+\underline{X Y}|^{2 n}}, \quad K \mid(\underline{X}, \underline{Y})=-\frac{1}{\omega_{2 n}} \frac{1+\underline{X}|\underline{Y}|}{\left.|1+\underline{X}| \underline{Y}\right|^{2 n}},
$$

for arbitrary $\underline{X} \neq \underline{Y}$, where $\omega_{2 n}$ denotes the area of the unit sphere $S^{2 n}$.

Theorem 4.1 For arbitrary $\underline{\mathcal{S}}_{\underline{X}}(\underline{Y}), \underline{X} \in B(1), \underline{Y} \in S^{2 n}$, the reproducing Szegö kernel has the expression

$$
\underline{\mathbf{S}}_{\underline{X}}(\underline{Y})=\left(\begin{array}{cc}
\mathcal{K} & \mathcal{K}^{\dagger} \\
\mathcal{K}^{\dagger} & \mathcal{K}
\end{array}\right)
$$

where $\mathcal{K}=-(K+i K \mid), \quad \mathcal{K}^{\dagger}=K-i K \mid$.

Proof Noticing, for arbitrary $\underline{Y}, \underline{Y} \mid \in S^{2 n}$,

$$
\begin{aligned}
& K(\underline{X}, \underline{Y})=-\frac{1}{\omega_{2 n}} \frac{1+\underline{X Y}}{|1+\underline{X Y}|^{2 n}}=\frac{1}{\omega_{2 n}} \frac{\underline{\bar{Y}}-\underline{\bar{X}}}{|\underline{Y}-\underline{X}|^{2 n} \underline{Y},} \\
& K\left|(\underline{X}, \underline{Y})=-\frac{1}{\omega_{2 n}} \frac{1+\underline{X}|\underline{Y}|}{\left.|1+\underline{X}| \underline{Y}\right|^{2 n}}=\frac{1}{\omega_{2 n}} \frac{\underline{Y}|-\underline{X}|}{|\underline{Y}|-\left.\underline{X}\right|^{2 n}} \underline{Y}\right|,
\end{aligned}
$$

we have

$$
\left(\begin{array}{cc}
\mathcal{K} & \mathcal{K}^{\dagger} \\
\mathcal{K}^{\dagger} & \mathcal{K}
\end{array}\right)=\left(\begin{array}{rr}
-E+i E \mid & E-i E \mid \\
E-i E \mid & -E+i E \mid
\end{array}\right) \frac{1}{2}\left(\begin{array}{rr}
Y+Y \mid & -Y+Y \mid \\
-Y+Y \mid & Y+Y \mid
\end{array}\right) .
$$

Then we get

$$
\mathbf{D}_{\left(\underline{Z}, \underline{Z}^{\dagger}\right)} \underline{\mathbf{S}}_{\underline{X}}(\underline{Y})=\mathbf{0}, \underline{X} \in B(1) .
$$

Applying the Cauchy formula in $[20,26]$, for arbitrary $\mathbf{G}_{2}^{1}(\underline{Y}) \in \mathbf{L}_{2}\left(S^{2 n}\right)$, we have

$$
\mathbf{G}_{2}^{1}(\underline{X})=\int_{S^{2 n}} \underline{\mathbf{S}}_{\underline{X}}(\underline{Y}) \mathbf{G}_{2}^{1}(\underline{Y}) d S_{\underline{Y}}, \underline{X} \in B(1) .
$$

Remark 4.2 Let $\mathbb{S}=\mathbb{C}_{n} \mathcal{I}$ denote the spinor space, where $\mathcal{I}=\mathcal{I}_{1} \ldots \mathcal{I}_{n}$ is the primitive identity element with $\mathcal{I}_{j}=\mathfrak{f}_{j} \mathfrak{f}_{j}^{\dagger}=\frac{1}{2}\left(1-i e_{j} e_{n+j}\right), \mathbb{S}=\bigoplus_{j}^{n} \mathbb{S}_{j}$ with $\mathbb{S}_{j}=\left(\mathbb{C} \wedge_{n}^{\dagger}\right)^{(j)} \mathcal{I}$, and the Grassmann algebra $\left(\mathbb{C} \wedge_{n}^{\dagger}\right)^{(j)}, j=1,2, \ldots, n$ generated by the Witt basis $\left\{\mathfrak{f}_{1}^{\dagger}, \ldots, \mathfrak{f}_{n}^{\dagger}\right\}$ (see Ref. e.g. [26]). When $\mathbf{G}_{0}=\left(\begin{array}{ll}g & 0 \\ 0 & g\end{array}\right)$ with $g$ taking values in the homogeneous n-space of spinor space $\mathbb{S}_{n}$, i.e., $g\left(z_{1}, \ldots, z_{n}\right)=g_{n}\left(z_{1}, \ldots, z_{n}\right) \mathfrak{f}_{1}^{\dagger} \ldots \mathfrak{f}_{n}^{\dagger} \mathcal{I}$ with $g_{n}\left(z_{1}, \ldots, z_{n}\right)$ being complex valued function defined in $\mathbb{R}^{2 n} \cong \mathbb{C}^{n}$, then by direct calculation, term (4.3) induces to the term as follows

$$
g_{n}(\underline{X})=\int_{S^{2 n}}(-1)^{\frac{n(n+1)+2}{2}} \frac{(n-1) !}{(2 \pi i)^{n}} \sum_{j=1}^{n} \frac{v_{j}^{c}-z_{j}^{c}}{|v-z|^{2 n}} \widehat{d v_{j}^{c}} g_{n}(\underline{Y}) .
$$

This implies that the matrix Szegö kernel $\underline{\mathbf{S}}_{\underline{X}}(\underline{Y})$ reduces to the Martinelli-Bochner kernel

$$
(-1)^{\frac{n(n+1)+2}{2}} \frac{(n-1) !}{(2 \pi i)^{n}} \sum_{j=1}^{n} \frac{v_{j}^{c}-z_{j}^{c}}{|v-z|^{2 n}}
$$


of holomorphic functions of several complex variables on the unit ball, where

$$
\left(v_{1}, \ldots, v_{n}\right) \in\left\{\left(v_{1}, \ldots, v_{n}\right): \sum_{j=1}^{n}\left|v_{j}\right|^{2}=1\right\},\left(z_{1}, \ldots, z_{n}\right) \in\left\{\left(z_{1}, \ldots, z_{n}\right): \sum_{j=1}^{n}\left|z_{j}\right|^{2}<1\right\} .
$$

Furthermore, if $n=1$, term (4.5) is equal to the case

$$
\frac{1}{2 \pi i} \frac{1}{v-z}
$$

where $v \in\{v \in \mathbb{C}:|v|=1\}, z \in\{z \in \mathbb{C}:|v|<1\}$, which is the Szegö kernel for analytic functions of one complex variable on the disk.

As an application of the matrix Szegö kernel, we get the theorem as follows.

Theorem 4.3 If $\mathbf{G}_{2}^{1} \in \mathbf{L}_{2}\left(S^{2 n}\right)$, then the solution of the system (3.1) is explicitly formulated by

$$
\mathbf{F}_{2}^{1}(\underline{X})=\int_{S^{2 n}}\left(\underline{\mathbf{S}}_{\underline{X}}(\underline{Y})+\left.\underline{\nu}\right|_{S^{2 n}} \underline{\mathbf{S}}_{\underline{X}}(\underline{Y})\right) \mathbf{G}_{2}^{1}(\underline{Y}) d S_{\underline{Y}},
$$

where $\underline{\mathbf{S}}_{X}(\underline{Y})$ as $(4.1)$ and $\left.\underline{\nu}\right|_{S^{2 n}}$ as Remark 3.5.

Proof Applying Lemma 3.3, associating with Theorem 3.11, it follows the result.

Remark 4.4 Hereby, we only present the explicit solution of the classical Dirichlet problem on the ball of higher-dimensional space, by means of the matrix Szegö kernel. In fact, this leads the decomposition of the classical matrix Poisson kernel by

$$
\left(\begin{array}{ll}
P(\underline{X}, \underline{Y}) & 0 \\
0 & P(\underline{X}, \underline{Y})
\end{array}\right)=\underline{\mathbf{S}}_{\underline{X}}(\underline{Y})+\left.\underline{\nu}\right|_{S^{2 n}} \underline{\mathbf{S}}_{X}(\underline{Y}),
$$

where

$$
P(\underline{X}, \underline{Y})=\frac{1}{\omega_{2 n}} \frac{1-|\underline{X}|^{2}}{|\underline{X}-\underline{Y}|^{2 n}}, \underline{X} \neq \underline{Y} .
$$

Following the same argument, we could consider the Dirichlet problem for matrix functions on the general sub-domains of higher-dimensional space, which will be discussed in the forthcoming paper.

\section{References}

[1] G. Szego, Über orthogonale polynome, die zu einer gegebenen Kurve der komplexen Ebene gehören. Math. Z., 1921, 9: 218-270

[2] S. Bell, The Cauchy transform, potential theory and conformal mapping. CRC Press, Boca Roton, 1992

[3] N. Kerzman, E. M. Stein, The Cauchy kernel, the Szegö kernel and the Riemann mapping function. Math. Ann., 1971, 236: 85-93

[4] S. Bell, Solving the Dirichlet problem in the plane by means of the Cauchy integral. Indiana Univ. Math. Journal, 1990,39(4): 1355-1371

[5] S. Bell, The Szegö projection and the classical objects of potential theory in the plane. Duke Math. Journal, 1991, 64(1): 1-26

[6] S. Bernstein, L. Lanzani, Szegö projections for Hardy spaces of monogenic functions and applications. Int. J. Math. Math. Sci., 2002,29: 613-624

[7] D. Calderbank, Clifford analysis for Dirac operators on manifolds with boundary. Max-Planck-Institute für Mathematik, Bonn, 1996

[8] R. Delanghe, On some properties of the Hilbert transform in Euclidean space. Bull. Belg. Math. Soc. Simon Stevin, 2004, 11: 163-180 
[9] D. Constales, R. S. Krausshar, Szegö and polymonogenic Bergman kernels for half-space and strip domains, and single-periodic functions in Clifford analysis. Complex Var. Elliptic Equ., 2002, 47(4): 349-360

[10] R. Delanghe, F. Brackx, Hypercomplex function theory and Hilbert modules with reproducing kernel. Proc. London Math. Soc., 1978, 37(3): 545-576

[11] J. Ryan, Complexified Clifford analysis. Complex Var. Theory Appl., 1982,1(1): 119-149

[12] F. Brackx, R. Delanghe, F. Sommen, Clifford Analysis, London: Pitman, 1982

[13] R. Delanghe, F. Sommen, V. Souček, Clifford analysis and spinor valued functions. Dordrecht: Kluwer Academic Publishers, 1992

[14] K. Gürlebeck, W. Sprössig, Quaternionic and Clifford calculus for physicists and engineers. Wiley: Chichester, New York, 1997

[15] J. E. Gilbert, M. A. M. Murry, Clifford algebra and Dirac operators in harmonic analysis. Cambridge Studies in Advances Mathematics 26, Cambridge University Press, Cambridge, 1991

[16] A. McIntosh, Clifford algebras, Fourier transforms, and singular convolution operators on Lipschitz surfaces. Rev. Mat. Iberoamericana, 1994,10(3): 665-721

[17] M. Ku, J.Y. Du, On integral representation of spherical k-regular functions in Clifford analysis. Adv. Appl. Clifford Alg., 2009,19(1): 83-100

[18] M. Ku, Integral formula of isotonic functions over unbounded domain in Clifford analysis. Adv. Appl. Clifford Alg., 2010,20(1): 57-70

[19] M. Ku, J. Y. Du, D.S. Wang, On generalization of Martinelli-Bochner integral formula using Clifford analysis. Adv. Appl. Clifford Alg., 2010,20(2): 351-366

[20] F. Brackx, et al., Fundaments of Hermitean Clifford analysis. I. Complex structure. Complex Anal. Oper. Theory, 2007, 1: 341-365

[21] F. Brackx, et al., Fundaments of Hermitean Clifford analysis. II. splitting of $h$-monogenic equations. Complex Var. Elliptic Equ., 2007,52(10-11): 1063-1079

[22] R. Rocha-Chavez, M. Shapiro, F. Sommen, Integral theorems for functions and differential forms in $\mathbb{C}_{m}$. Research Notes in Mathematics 428, Chapman Hall/CRC, New York, 2002

[23] F. Brackx, H. De Schepper, F. Sommen, The Hermitian Clifford analysis toolbox. Adv. Appl. Clifford Alg., 2008,18: 451-487

[24] F. Brackx, B. De Knock, H. De Schepper, F. Sommen, On Cauchy and Martinelli-Bochner integral formulae in Hermitean Clifford analysis. Bull. Braz. Math. Soc., 2009,40(3): 395-416

[25] F. Brackx, B. De Knock, H. De Schepper, A matrix Hilbert transform in Hermitean Clifford analysis. J. Math. Anal. Appl., 2008,344: 1068-1078

[26] M. Ku, D.S. Wang, Half Dirichlet problem for matrix functions on the unit ball in Hermitean Clifford analysis. J. Math. Anal. Appl., 2011,374: 442-457

[27] R. Abreu Blaya, J. Bory Reyes, T. Moreno García, Hermitian decomposition of continuous functions on a fractal surface. Bull. Braz. Math. Soc., 2009,40(1): 107-115

[28] R. Abreu Blaya, J. Bory Reyes, F.Brackx, B. De Knock, H. De Schepper, D. Peña Peña, F. Sommen, Hermitean Cauchy integral decomposition of continuous functions on hypersurfaces. Boundary Value Problems, 2008, Article ID 57: 425256, doi:10.1155

[29] D. Eelbode, F.L. He, Taylor series in Hermitean Clifford analysis. Complex Anal. Oper. Theory, 2011,5(1): 97-111

[30] M. Ku, U. Kähler, D. S. Wang, Half Dirichlet problem for the Hölder continuous matrix functions in Hermitian Clifford analysis. Complex Var. Elliptic Equ., 2013,58(7): 1037-1056 